\title{
Research on Phased Array Radar Resource Management in Searching Mode
}

\author{
Junqing. Wu , Ting. Cheng \\ School of Electronic Engineer, UESTC, 611731 Chengdu, China
}

\begin{abstract}
Multifunction phased array radar, with rational management of time resource and energy resource, can search and track multiple targets simultaneously. A resource management optimization model for phased array radar in searching mode has been proposed, whose objection is to minimize the total resource consumption under the guarantee of searching performance with controllable parameters: search frame period, dwell time and signal duty circle. Both radial cumulative detection probability and tangential cumulative detection probability are considered in the searching performance. The consideration of tangential cumulative detection probability as one of the constraints is the main contribution here. The solution to this model by applying genetic algorithm suggests that this model is more suitable in actual scene.
\end{abstract}

\section{Introduction}

In modern battlefield environment, target characteristics and attack warfare have become increasingly complex, and make the continuous extension of radar functional requirements. The less resource the searching task consumes, the more resource can be used for other tasks, which can obtain a better performance in target detecting and tracking.

The resource management [1] in searching mode is actually the parameter control of the searching tasks. Billiam[2][3] obtained the optimal search frame period when there is no high prior load in radar system, which not only can meet the tracking initiation condition, but also consume the least radar resources. Based on this, Jianbin Lu [4] derived optimal searching model of multifunction phased array radars with limited searching resources and proposed the criterion for the optimum parameter choice of search frame period and detection range in detail. Litao Zhang studied searching parameters of phased array radar from the point of view of concept and system and presented the selection principle and method of a number of searching parameters [5]. Reference [6] discussed the influence on searching performance caused by the dwell time of a searching beam, scan time and false alarm probability, then designed the optimal operation parameters for searching performance. Above research only focus on the situation when target fly to the target directly, only the racial cumulative detection probability is considered. However, because of the limit of the angle range of searching area, target may cross the angle border at a short distance with both radial and tangential velocity. However, the target has both racial and tangential speed; the tangential detection performance should not be omitted.

A resource management algorithm for phased array radar in searching mode is presented, which sets the search frame period, dwell time, signal duty circle as controllable parameters to minimize the total recourse consume under the guarantee of searching performance. Resources include time resource and energy resource. The searching performance can be indicated in tangential cumulative detection probability and radial cumulative detection probability. Here, we consider the velocity of target have both radial and tangential velocity.

This paper is organized as follows. The resource management optimization model in searching mode is formulated Section II. In Section III we utilize Genetic Algorithm to solve this optimization problem. The simulation result is presented in Section IV. Section V is the conclusion.

\section{Resource management optimization model in searching}

The criterion for the optimum choice of parameter is the value which will give the required searching performance with minimum resource consumption. Considering a phased array radar which is performing the searching task, the controllable parameters include search frame period $T_{f}$, dwell time $t_{B}$ and signal duty circle $\eta$. First of all, we formulate the relationship between controllable parameters and resource consumption..

\subsection{Objective function}


Let $\eta, t_{B}, T_{f}$ be the parameters of searching task in the surveillance space. Search frame period $T_{f}$ and dwell time $t_{B}$ reflect the time resource consumption, $\eta$ indicates the energy resource consumption. Considering the time and energy resource consumption synthetically, the objective function can be calculated as,

$$
\min _{\left(\eta, t_{B}, T_{f}\right)} c_{1} \frac{t_{B} N_{B}}{T_{f}}+c_{2} \eta
$$

where $N_{B}$ is the number of dwell beams in searching area. The values of $c_{1}, c_{2}$ show the importance of different resources.

\subsection{Constraint conditions}

Here, the relationship between controllable parameters and searching performance is formulated. That is, we formulate the constraint conditions. In [2], searching performance is defined in terms of the track formation range. It supposed that the velocity of the target only have a radial component. However, in actual scene, target approach also with a tangential velocity component. Therefore, similarly to the concept of track formation range, the track formation angle is defined in this paper. We use these two indices to describe the searching performance.

Firstly, we show the case that target approach to radar only with radial velocity. Donate the largest operational range provided by radar system as $R_{s}$. Assume the target is approaching with a radial velocity of $v$, track formation range is $R_{t}$. Thus the radial cumulative detection probability 2 is

$$
\begin{aligned}
& P_{d}\left(R_{t}\right)= \\
& \frac{1}{\Delta r} \int_{R_{s}-\Delta r}^{R_{s}}\left\{1-\left\lceil\prod_{i=0}^{\left\lceil\frac{R_{s}-R_{t}}{\Delta r}\right\rceil}\left[1-P_{d c} \cdot P_{d i}(r-i \Delta r)\right]\right\} d r\right.
\end{aligned}
$$

where $\lceil$.$\rceil means rounding down to the nearest integer,$ $\Delta r=v T_{f}$ is the radial range during $T_{f} . P_{d c}$ is the probability of successful confirmation, $P_{d i}(r-i \Delta r)$ is the single detection probability at range $r-i \Delta r$, which is related to the signal to noise ratio.

$$
P_{d i}=P_{f a}^{\frac{1}{1+\mathrm{SNR}_{i}}}
$$

where $P_{f a}$ is the probability of false alarm. According to the radar function 7 , signal to noise ratio can be written as

$$
S N R_{i}=\frac{P_{t} \eta G_{t} G_{r} \lambda^{2} \sigma t_{B}}{(4 \pi)^{3}\left(r-i v T_{f}\right)^{4} k T_{0} F_{n} L_{t o t}}
$$

In order to guarantee the searching performance, the radial cumulative detection probability has to reach a certain threshold $P_{D}$.Then

$$
P_{d}\left(R_{t}\right) \geq P_{D}
$$

Secondly, we analyze the case which target approach to radar only with tangential velocity. Donate the angle searching range as $\left[\theta_{1}, \theta_{2}\right]$.Assume the target is approaching with a tangential velocity of $v_{x}$, track formation angle is $\theta_{t}$,

$$
\theta_{t}=\left(\theta_{2}-\theta_{1}\right) Q, Q \in(0,1)
$$

That is to say, when target fly from angle $\theta_{1}$ to $\theta_{s}, \theta_{s}=\theta_{1}+\theta_{t}$, the tangential cumulative detection probability should reach a certain threshold to guarantee the searching performance. Fig. 1 shows a visualization definition of track formation angle. Suppose that the target fly into the searching area at time $t_{0}$, and fly to angle $\theta_{s}$ at time $t_{\max }$.

$$
t_{\max }=t_{0}+\frac{\theta_{t} R_{x}}{v_{x}}
$$

$R_{x}$ is the radial range when target fly into searching area along tangential direction.

Now calculate the tangential cumulative detection probability during $\left[t_{0}, t_{\max }\right]$. Fig. 2 shows the changes of radar beam pointing and target's direction with time. In fig.2, bold line describes the relation of radar beam pointing to time. This is a periodic function of $T_{f}$.

To a certain beam, the range of beam pointing is $\left[B_{w}(i-1)+\theta_{1}, B_{w}(i)+\theta_{1}\right]$ during the time interval $\left[T_{f}(j-1)+(i-1) t_{B}, T_{f}(j-1)+i t_{B}\right]$.The dash dot line represents the target angle position changing with time which can be expressed as

$$
\theta_{t}(t)=\frac{v_{x}\left(t-t_{0}\right)}{r_{x}}+\theta_{1}
$$

The intersections of these two lines represent that the radar illuminates the target. But the dwell time on the target is different in different encounter time. Here, just call the dwell time on target the real dwell time. 


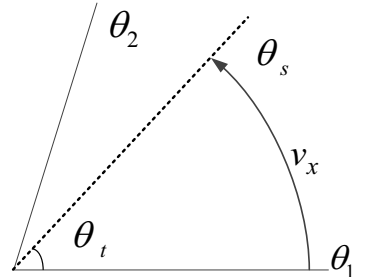

Figure 1. Visualized definition of track formation angle.

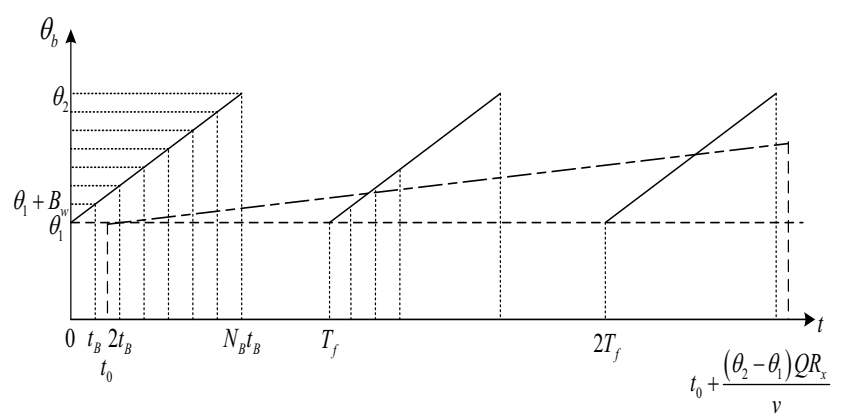

Figure 2. Changes of radar beam pointing and target's direction with time

As the total number of searching beam is $I$ during $\left[t_{0}, t_{\max }\right]$, then we can obtain the tangential cumulative detection probability as,

$$
\prod_{n_{\text {count }}=1}^{I}\left[1-P_{d c} \cdot P_{d}\left(t_{B}^{\prime}\left(t_{0}\right), n_{\text {count }}\right)\right]
$$

Where

$$
\begin{gathered}
I=I_{1}+I_{2} \\
I_{1}=\left\lceil\frac{\left(t_{\text {max }}-t_{0}\right)}{T_{f}}\right\rceil * N_{B}=\left\lceil\frac{\theta_{\text {max }} r_{x}}{v_{x} T_{f}}\right\rceil * N_{B} \\
I_{2}=\min \left[\bmod \left(\frac{\left(t_{\text {max }}-t_{0}\right)}{T_{f}}\right) * T_{f} / t_{B}, N_{B}\right]
\end{gathered}
$$

Where $t_{B}{ }^{\prime}$ can be calculated as follows.

Supposing at $t_{0}$ moment, target fly into the searching area with angle position $\theta_{1}$.At this time, the serial number of dwell beam is $n_{0}$.

$$
n_{0}=\left\{\begin{array}{l}
\left\lfloor\frac{t_{0}}{t_{B}}\right\rfloor, \frac{t_{0}}{t_{B}}<\mathrm{N}_{B} \\
0, \quad \frac{t_{0}}{t_{B}}>\mathrm{N}_{B}
\end{array}\right.
$$

Then start the beam counter $n_{\text {count }}$, which is started from 0 and finished in $I-1$. So to the $n_{\text {count }}$-th beam, the counter of search frame period $n_{T_{f}}$ is

$$
n_{T_{f}}=\left\lfloor\frac{n_{0}+n_{\text {count }}}{N_{B}}\right\rfloor
$$

The radar beam serial number $n_{\text {search }}\left(n_{\text {search }} \geq 1\right)$ is

$$
n_{\text {search }}= \begin{cases}N_{B}, \quad n_{0}+n_{\text {count }}=k N_{B}, \\ \bmod \left(\frac{n_{0}+n_{\text {count }}}{N_{B}}\right), & \text { else, }\end{cases}
$$

where $k=1,2, \ldots$ The beam pointing is

$$
\left[\theta_{s 1}, \theta_{s 2}\right]=\left[\theta_{1}+\left(n_{\text {search }}-1\right) B_{w}, \theta_{1}+n_{\text {search }} B_{w}\right]
$$

The beam acting time is

$\left[t_{s 1}, t_{s 2}\right]=$

$\left[\left(n_{T_{f}}-1\right) T_{f}+\left(n_{\text {search }}-1\right) t_{B},\left(n_{T_{f}}-1\right) T_{f}+n_{\text {search }} t_{B}\right]$

Assuming in the moment interval $\left[\tilde{t}_{1}, \tilde{t}_{2}\right]$, target is illuminated by radar, so its beam position is between $\left[\theta_{s_{1}}, \theta_{s_{2}}\right]$. The $\left[\tilde{t}_{1}, \tilde{t}_{2}\right]$ can be solved by

$$
\theta_{s_{1}}=\frac{v\left(\tilde{t}_{1}-t_{0}\right)}{r}+\theta_{1}, \theta_{s_{2}}=\frac{v\left(\tilde{t}_{2}-t_{0}\right)}{r}+\theta_{1}
$$

If $\left[t_{s_{1}}, t_{s_{2}}\right]$ and $\left[\tilde{t}_{1}, \tilde{t}_{2}\right]$ intersect, the real dwell time $t_{B}{ }^{\prime}$ is the length of intersection. If the intersection is empty, the target is not illuminated. That is, $t_{B}{ }^{\prime}=0$ and $P_{d}\left(t_{B}^{\prime}\right)=0$.

However, the calculation above supposes that $t_{0}$ is known. In actual scene, it is a random variable, which is assumed to be uniform in general case. Therefore, the statistical tangential cumulative detection probability can be written as

$$
\begin{aligned}
& P_{d}\left(\theta_{t}\right)= \\
& \frac{1}{T_{f}} \int_{0}^{T_{f}}\left\{1-\prod_{n_{\text {count }}=1}^{I}\left[1-P_{d c} \cdot P_{d}\left(t_{B}{ }^{\prime}(t), n_{\text {count }}\right)\right]\right\} d t
\end{aligned}
$$

Furthermore, we have time constraint,

$$
\frac{N_{B} t_{B}}{T_{f}} \leq 1
$$


This means the time use factor should less than 1.

So the resource management optimization model of phased radar in searching mode can be given by,

$$
\begin{aligned}
& \min _{\left(\eta, t_{B}, T_{f}\right)} c_{1} \frac{t_{B} N_{B}}{T_{f}}+c_{2} \eta \\
& \text { s.t. }\left\{\begin{array}{l}
P_{d}\left(R_{t}\right) \geq P_{D} \\
P_{d}\left(\theta_{\max }\right) \geq P_{D} \\
\frac{N_{B} t_{B}}{T_{f}} \leq 1
\end{array}\right.
\end{aligned}
$$

\section{Genetic algorithm solving optimization model}

As described in Eq.21 the formulated resource management optimization model is nonlinear. Genetic algorithm [8] is one kind of adaptive random searching algorithms that simulate the evolution process and mechanism in nature to solve the optimization problems, it can automatically gain and simulate the knowledge about search-space, therefore, find the best solution. Based on the genetic algorithm, the resource management algorithm for phased radar is obtained. Fig. 3 shows the flow chart of it.

The value of objective function is considered as the adaptive value of genetic algorithm. The smaller the value of objective function is, the greater the adaptive value can be. If there is no individual to meet all the constraints in the initial population, we can continue to crossover and mutate to rebuild a new population until appropriate individual appears. When the evolutional generation has approached its maximal value, the algorithm ends. Otherwise the reorganized population will continue circulating.

\section{Simulation}

Consider a uniformly linear array with $M=256$, where the unit gap is the half of the wavelength. Here, constant parameters include wavelength $\lambda=5.45 \mathrm{~cm}$, Boltzmanns constant $k=1.38 \times 10^{-23} J / K$, standard temperature $T=230 K$, noise figure $F=2$, and the effective area duty ratio of antenna $\eta_{e}=0.5$. The angle range of the searching section is $\left[-40^{\circ}, 40^{\circ}\right]$, the largest operational range is $R_{s}=200 \mathrm{~km}$.In case of Swelling-I, set the average RCS of target to be $1 \mathrm{~m}^{2}$, the probability of false alarm to be $P_{f a}=10^{-6}$, the threshold of $P_{D}=0.95$.The radial velocity and tangential velocity are the same as $v_{r}=v_{t}=1.5 \mathrm{Ma}$. The weighting of different resource are $c 1=0.5, c 2=0.5$. Signal duty circle changes in $\eta \in[0,0.25]$, dwell time changes in
$t_{B} \in[0,26] m s$ and search frame period is chosen in $T_{f} \in[0,20] \mathrm{s}$. In genetic algorithm, the number of individuals in population is 1000 , the maximal number of

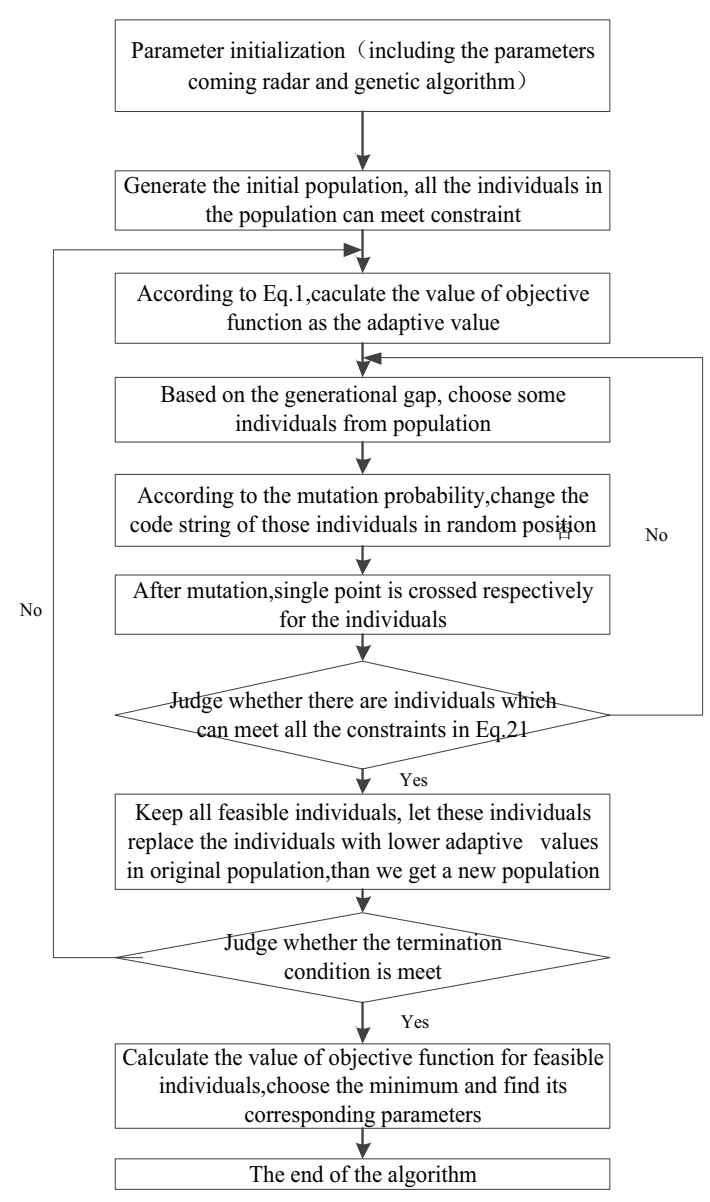

Figure 3. The flow chart of genetic algorithm

generations is 300 , the crossover probability is 0.7 , and the mutation probability is 0.05 , its generational gap is 0.95 . Firstly, we only considered the radial cumulative detection probability and time constriction. Fig.4 to Fig.6 are the changing curves of the dwell time, the search frame period and the signal duty circle respectively. When track formation range is small, radar can accumulate many times, in other words, the desired single detection probability is reduced. With the increase of track formation range, in order to meet the requirement of radial cumulative detection probability, the search frame should be smaller. In Fig.4, the dwell time increased before $120 \mathrm{~km}$, and then keeps the maximum. Fig. 5 shows the changing tendency of search frame period. When track formation range is larger than $120 \mathrm{~km}$, search frame period becomes smaller to increase the accumulation times. The signal duty circle increase just as Fig. 6 shows.

Then, use these optimal parameters values to calculate the tangential cumulative detection probability. Suppose the $\mathrm{Rx}=30 \mathrm{~km}, \mathrm{Q}=0.3$. The result is showing in Fig.7.

As we can see from Fig.7.,the tangential cumulative detection probability is less than 0.95 before $150 \mathrm{~km}$, so above parameters allocation cannot guarantee the required searching performance. So we should consider all the constraint conditions, including the radial 
cumulative detection probability, tangential cumulative detection probability and time limit.

In actual scene, the track formation range and track formation angle is always known. However, the radial range when target fly into searching area along tangential direction is a random variable. So the following results are the optimal parameters in different $R_{x}$. Here, we suppose $R_{t}=140 \mathrm{~km}, Q=0.3$. Fig. 8 to Fig.10 are the changing curves of the dwell time, the search frame period and the signal duty circle respectively. Parameters fluctuated at certain value in a small range. From the result we can see, $R_{x}$ doesn't have great influences when $R_{x}$ is larger than $40 \mathrm{~km}$. So we choose dwell time as $26 \mathrm{~ms}$, the search frame period as $14.7 \mathrm{~s}$, and signal duty circle as $22.3 \%$. However, when $R_{x}$ is less than $40 \mathrm{~km}$, the dwell time is less than $24 \mathrm{~ms}$, and the search frame period is less than 13s.So in order to meet the required searching performance, we choose dwell time as $24 \mathrm{~ms}$, the search frame period as $8.2 \mathrm{~s}$, and signal duty circle as $21.3 \%$ when $R_{x}$ is less than $40 \mathrm{~km}$.

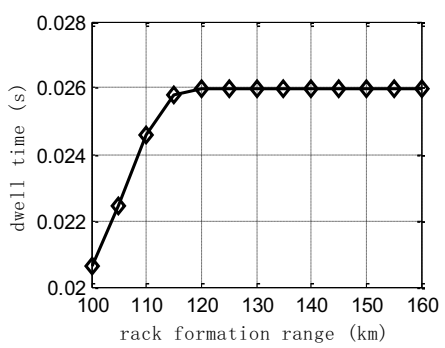

Fig.4. The relationship between dwell time and Rt

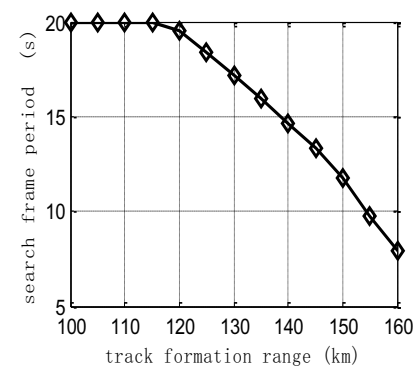

Fig.5. The relationship between search frame period and Rt
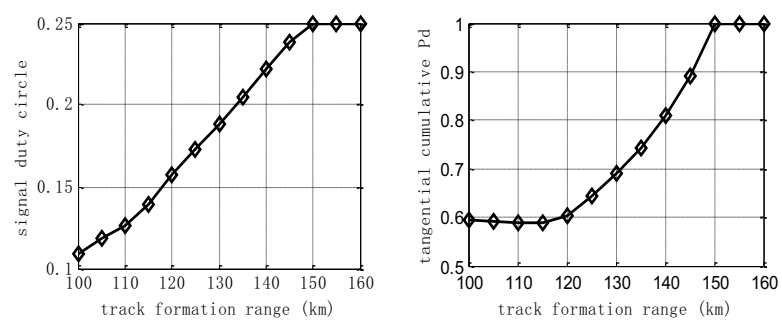

Fig.6. The relationship between signal duty circle and Rt(left)

Fig.7 The relationship between tangential cumulative detection probability and track formation range (right)
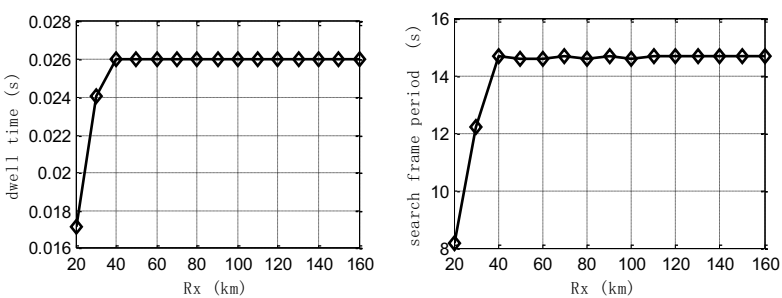

Fig. 8. The relationship between dwell time and Rx (left) Fig.9. The relationship between search frame period and Rx(right)

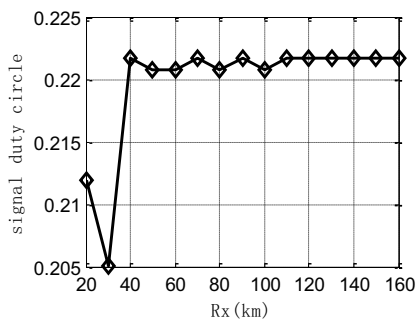

Fig.10. The relationship between signal duty circle and Rx

\section{Conclusions}

The resource management of phased array radar in searching mode is vital for the multifunction of it. An optimal mode is formulated whose objection is to minimize the total recourse consumption and constraint conditions include tangential cumulative detection probability, radial cumulative detection probability and time limit. The consideration of tangential cumulative detection probability is the main contribution here. The calculation of the tangential cumulative detection probability is given in detail. The constraint considered in this model can guarantee a more reliably searching performance. Simulation results demonstrate the necessity of the tangential constraint and the effectively of the resulting resource management algorithm

\section{References}

1. Michael Zatman. "Radar Resource Management for UESA,”. IEEE International Conf, 2002, 73-76

2. E. R. Billam, "Parameter optimisation in phased array radar," in Real-Time Management of Adaptive Radar Systems, IEE Colloquium on, London, 1992, pp. 34-37.

3. E. R. Billam, "Design and performance considerations in modern phased array radar," IEE Colloquium on, London, 1982, pp. 15-19.

4. J. B. Lu, W. D. Hu, and W. X. Yu, "Research on optimal search performance of phased array radars with limited resources," Systems Engineering and Electronics, Vol.26, No.10, pp. 1388-1390, May 2004.

5. L. T. Zhang, D. Li, and G. Y. Wang, "A study on search parameters in phased radar," Modern Radar, Vol.30, No.10, pp. 20-25, October 2008.

6. H. R. Zhang, H. W. Yang, and W. X. Yu, "Design of optimal search operation parameters for phased array radar," Acta Armamentarii, vol. 33, no. 9, pp. 1062-1065, Sep.2012. 
7. M. Y. Zhang, X. G. Wang, Radar Systems, Beijing: Publishing House of Electronics Industry, $4^{\text {nd }}, 2013$, ch6.

8. Holland J H. Adaption in Natural and Artificial Systems. Ann Arbor, USA: University of Michigan Press, 1975. 